\title{
The nature and duration of adaptation following long-term odor exposure
}

\author{
PAMELA DALTON and CHARLES J. WYSOCKI \\ Monell Chemical Senses Center, Philadelphia, Pennsylvania
}

\begin{abstract}
Any individual living or working in an odorous environment can experience changes in odor perception, some of which are long lasting. Often, these individuals report a significant reduction in the perception of an odor following long-term exposure to that odor (adaptation). Yet, most experimental analyses of olfactory adaptation use brief odorant exposures which may not typify real-world experiences. Using a procedure combining long-term odor exposure in a naturalistic setting with psychophysical tests in the laboratory, we present evidence to show that reduced odor intensity following long-term exposure is accompanied by odorant-specific shifts in threshold. Subjects were exposed continuously to one of two odorants while in their home for a period of 2 weeks. Exposure produced an odorant-specific reduction in sensitivity and perceived intensity compared with preexposure baselines: Detection thresholds for the adapting odorant were elevated following exposure and perceived intensity ratings for weak concentrations were reduced. For most individuals, reduced sensitivity to the test odorant was still evident up to 2 weeks following the last exposure. The persistence of the change, as evidenced by the duration of recovery from adaptation, distinguishes this phenomenon from the adaptation seen following shorter exposures and highlights the need for the study of exposure durations that are more similar to real-world exposures.
\end{abstract}

The decrease in responsiveness that occurs following continuous stimulation (adaptation) is a feature common to all sensory systems, including olfaction. In situations where information is provided by dynamic sources of sensory input, unvarying stimuli often cease to be detected or perceived at the original intensity. ${ }^{1}$

This phenomenon can be readily observed in situations where ambient odors are chronically present. Individuals who live or work in such an odorous environment often report that, with continued exposure, their perception of the ambient odor intensity is greatly reduced. Moreover, the perceptual changes that result from chronic exposure can be quite profound and durable. For example, it is commonly reported that following extended absences (hours to days) from the odorous environment, reexposure to the odor may still fail to elicit perception at the original intensity. At face value, this change seems to represent a very persistent type of adaptation. Yet, a review of numerous laboratory studies of olfactory adaptation (see Cometto-Muñiz \&

This research was supported by National Institutes of Health (NIH) Grant I F32 DC00197-01 to P.D., NIH Grant 5 RO1 DC002948-09 to C.J.W., and NIH Institutional Training Grant No. 5 T32 DC00014-17 to Monell Chemical Senses Center. The authors wish to thank Gary Beauchamp for his sustaining interest in the research question. We also thank Gary Beauchamp, J. W. Whitlow, Jr., and two anonymous reviewers for their invaluable comments on this manuscript. We are grateful to Robert Semanchik, formerly of Waterbury Co., Waterbury, $\mathrm{CT}$, for providing odorant delivery equipment and advice on its use, and Craig Warren of International Flavors and Fragrances, Union Beach, NJ, for providing advice and the odorants used in this research. Correspondence should be addressed to P. Dalton, Monell Chemical Senses Center, 3500 Market Street, Philadelphia, PA 19104 (e-mail: pdalton@pobox.upenn.edu).
Cain, 1995, for a recent summary) yielded no clear interpretation of the perceptual changes that follow longterm odor exposures or evidence of sensory adaptation as the basis for these changes.

The uncertainty arises because most research on olfactory adaptation (and studies of adaptation in sensory systems, generally) examines relatively transient changes in stimulus detection or perceived intensity. For example, in experiments on olfactory adaptation, the duration of exposure to the adapting stimulus (and hence the period of adaptation) has rarely exceeded several hours and is often far less (e.g., Berglund, 1977; Berglund, Berglund, Engen, \& Lindvall, 1971; Cain, 1970; Cain \& Polak, 1992; Ekman, Berglund, Berglund, \& Lindvall, 1967; Pierce, Wysocki, \& Aronov, 1993). Because olfactory adaptation can be produced with relatively short exposures, these durations are sufficient for investigating many parameters of the phenomenon. One sniff of an odorant can result in a significant decrease in the perceived odor intensity and continuous exposures of as little as $\mathbf{2 0}$ min have been sufficient to produce elevated detection thresholds. Recovery from adaptation proceeds rapidly: Once the odorant is removed, intensity judgments and detection thresholds often return to preexposure levels faster than the adaptation reaches asymptotic levels (see, e.g., Cain, 1974; Steinmetz, Pryor, \& Stone, 1970).

However, exposures to odors in natural environments often occur over far longer periods than have heretofore been investigated. Many individuals use the same fragranced products daily for weeks or months; others enter the same odorous workplace 5 days a week for many years. Changes in the detectability or perceived intensity of an odorant that occur after weeks or years of exposure 
may differ materially from the changes occurring after $5 \mathrm{~min}$, an hour, or even a single day. Several areas of investigation have been suggestive of this idea. Odorant exposures of up to $7 \mathrm{~h}$ have produced shifts in threshold or in perceived intensity that show only partial recovery from adaptation by 90 min following cessation of exposure (Andersen et al., 1983; Gagnon, Mergler, \& Lapare, 1994; Mergler \& Beauvais, 1992). In addition, numerous researchers have reported both general and specific olfactory dysfunction following chronic exposure to various chemicals in the workplace (Ahlström, Berglund, Berglund, Lindvall, \& Wennberg, 1986; Schwartz, Ford, Bolla, Agnew, \& Bleecker, 1991). ${ }^{2}$ Nonetheless, the nature of olfactory adaptation that develops in normal individuals following repeated or chronic odorant exposures remains largely unexplored under controlled conditions.

The adaptation produced by prolonged exposure to an odor may differ qualitatively from short-term olfactory adaptation. For example, electrophysiological studies in animals show that even brief pulses or short periods of odorant stimulation produce transient decrements in receptors in the olfactory epithelium, a process termed "receptor fatigue" (Ekblom, Flock, Hansson, \& Ottoson, 1984; Mozell, 1962; Potter \& Chorover, 1976); such changes may also be associated with desensitization of the chemoreceptive membrane (Getchell, 1986). Prolonged odor stimulation, however, could produce more durable response decrements, either through changes in the periphery or more persistent decrements in responsiveness in structures higher in the central nervous system (CNS) pathway (i.e., the olfactory bulb; Potter \& Chorover, 1976). Alternatively (or additionally), prolonged odor exposure may produce response decrements mediated by cognitive processes, such as attention and memory (Engen, 1982).

The present investigation focuses on assessing the characteristics of human olfactory adaptation resulting from prolonged exposure to an odorant. We examined the development of olfactory adaptation and recovery for two odorants over both short-term and long-term exposure durations. In the short-term experiment, we measured adaptation produced during 10-min exposures to an odorant. In the long-term experiment, we measured the development of adaptation over daily odorant exposures for a 2-week period. In the long-term experiment, we used field exposures in the home as a practical way to simulate environmental odor adaptation. These field exposures were combined with psychophysical assessments at weekly intervals to determine the effect of chronic exposure to an odorant on its detectability and perceived intensity. This research had three objectives: (1) to develop a method for exposing individuals to an odor for an extended period in order to study olfactory adaptation similar to that described in real-world contexts, (2) to document the extent and locus of any changes in detectability or perceived intensity following such exposures using traditional psychophysical measures of olfactory sensitivity and intensity, and (3) to compare subjects' perception of odor intensity in the home environment with sensitivity measured in the laboratory.

In Experiment 1 we investigated self-adaptation (a decrease in perceived intensity following exposure) and cross-adaptation (the decrease in perceived intensity following exposure to a different odor) for two perceptually distinctive odors (citrus and balsam) in a short-term adaptation paradigm. After establishing that exposure to each of these two odorants produced significant short-term self-adaptation (but not comparable cross-adaptation), we used the same odorants in Experiment 2 to measure the development and specificity of long-term adaptation and recovery on thresholds and judged intensity, produced by 2 weeks of daily odorant exposure.

\section{EXPERIMENT 1 Short-Term Adaptation and Cross-Adaptation}

A test of odorant-specific self-adaptation necessarily required measuring sensitivity to two odorants. One odorant was used as the adapting/exposure odorant for half of the subjects and the other odorant served as the control (to rule out general shifts in olfactory sensitivity or changes in criterion). A citrus odor (citralva, International Flavors \& Fragrances, Union Beach, NJ) and a balsam fir needle odor (iso bornyl acetate, International Flavors \& Fragrances, Union Beach, NJ) were chosen. These two odorants were selected to serve as test and control odorants for both studies on the basis of three criteria: (1) They were single-odor compounds, not mixtures; (2) they were perceptually dissimilar; and (3) they were both relatively pleasant and familiar. Although in the real world odorants are typically experienced in mixtures, the first criterion was included because most research on olfactory self-adaptation has been conducted with single odorants (but see Berglund \& Engen, 1993; Schiet \& Cain, 1990). The second criterion was used because perceptually similar compounds may be more likely than perceptually dissimilar compounds to show cross-adaptation (see, e.g., Cain \& Polak, 1992; Köster, 1971; Todrank, Wysocki, \& Beauchamp, 1991). The third criterion was included to facilitate compliance by subjects who agreed to live with this odorant in their home for 2 weeks. ${ }^{3}$

Because the primary goal of this investigation was to measure self-adaptation following long-term odorant exposure, it was important to establish that the chosen stimuli would self-adapt but not cross-adapt. If cross-adaptation occurred between the two odorants, exposure to one could produce a decrease in perceived intensity for both. This result could not be discriminated from a general decrease in olfactory sensitivity. Recently, a simple procedure for assessing the degree of both short-term self- and crossadaptation was developed by Pierce and his colleagues (Pierce et al., 1993). We briefly describe the procedure below. Readers are referred to the original paper for a full description of the method. 


\section{Method}

\section{Subjects}

Eight subjects ( 5 female, 3 male) were recruited from the University of Pennsylvania. Their mean age was 25.8 years. All subjects were paid for their participation.

\section{Stimuli}

A 12-step binary dilution series ranging from strong to weak was used for each odorant. The odorants were diluted in $10 \mathrm{ml}$ of odorless, light, white mineral oil and were presented in cleaned $270-\mathrm{ml}$ polypropylene bottles with plastic flip-top caps. The dilution series for citralva (MW $=149.2$ ) ranged from $1.19 \mathrm{M}$ (Step 0: $20 \% \mathrm{v} / \mathrm{v} ; 178 \mathrm{~g} / \mathrm{L}$ ) to $0.58 \mathrm{mM}$ (Step $11: 4 \times 10^{-3} \% \mathrm{v} / \mathrm{v} ; 0.086 \mathrm{mg} / \mathrm{L}$ ). The iso bornyl acetate series $(\mathrm{MW}=196.3)$ ranged from $2.51 \mathrm{M}$ (Step 0: $50 \% \mathrm{v} / \mathrm{v} ; 492 \mathrm{~g} / \mathrm{L}$ ) to $1.22 \mathrm{mM}$ (Step 11: $1.22 \times 10^{-2 \%}$; $0.240 \mathrm{mg} / \mathrm{L}$ ).

\section{Procedure}

All subjects were tested in two sessions, separated by a day, in which each odorant served as the adapting odorant. A forced-choice staircase procedure was used at the beginning of the session to equate the intensities of each odorant. For most subjects, Step 2 or Step 3 of citralva was judged as perceptually equivalent to the Step 2 concentration of iso bornyl acetate and was used as the testing stimulus. The next higher citralva concentration and Step 1 of iso bornyl acetate were used as the adapting stimuli.

After a 2-min rest, subjects rated the intensities of the iso bornyl acetate Step 2 stimulus and the matched citralva stimulus, using magnitude estimation. This provided a check on the matching procedure: If the estimates were different by more than $20 \%$ the matching procedure was repeated. This continued until magnitude estimates were in agreement with the intensity matching procedure and it could be concluded that the two stimuli were perceptually equivalent for that subject.

After the stimuli were matched, the adaptation phase was begun. Subjects were required to repeatedly sniff the adapting stimulus (either Step 1 of iso bornyl acetate or the next stronger step above the equivalent intensity of citralva). Every $15 \mathrm{sec}$ during the adaptation period, subjects received a test probe between sniffs of the adapting stimulus. The test probe, either iso bornyl acetate or citralva, alternated on sequential trials so that subjects made a total of 20 ratings ( 10 iso bornyl acetate, 10 citralva) during the 5 -min adaptation period.

Following the adaptation period, subjects stopped sniffing the adapting odorant and simply made 10 ratings ( 5 iso bornyl acetate and 5 citralva) of the test probe. These ratings were made every $15 \mathrm{sec}$ during the period of recovery.

\section{Results}

Each magnitude estimate was converted to a proportion of the initial magnitude estimate for each odorant. Figure 1 shows these estimates averaged across all 8 subjects (each subject contributed one observation on each test probe). Repeated measures analyses of variance (ANOVAs) were performed to ascertain whether estimates were significantly different from the initial estimate for the odor $(100 \%)$. Planned comparisons revealed that both odorants showed significant self-adaptation when estimates made during adaptation were contrasted to the original magnitude estimate [for citralva, $F(1,7)=$ $359.53, p<.0001$; for iso bornyl acetate, $F(1,7)=263.41$, $p<.0001$, respectively]. In contrast, we did not judge exposure to one odorant to produce cross-adaptation to the other at equivalent levels: When estimates made during adaptation were contrasted to the original magnitude es-
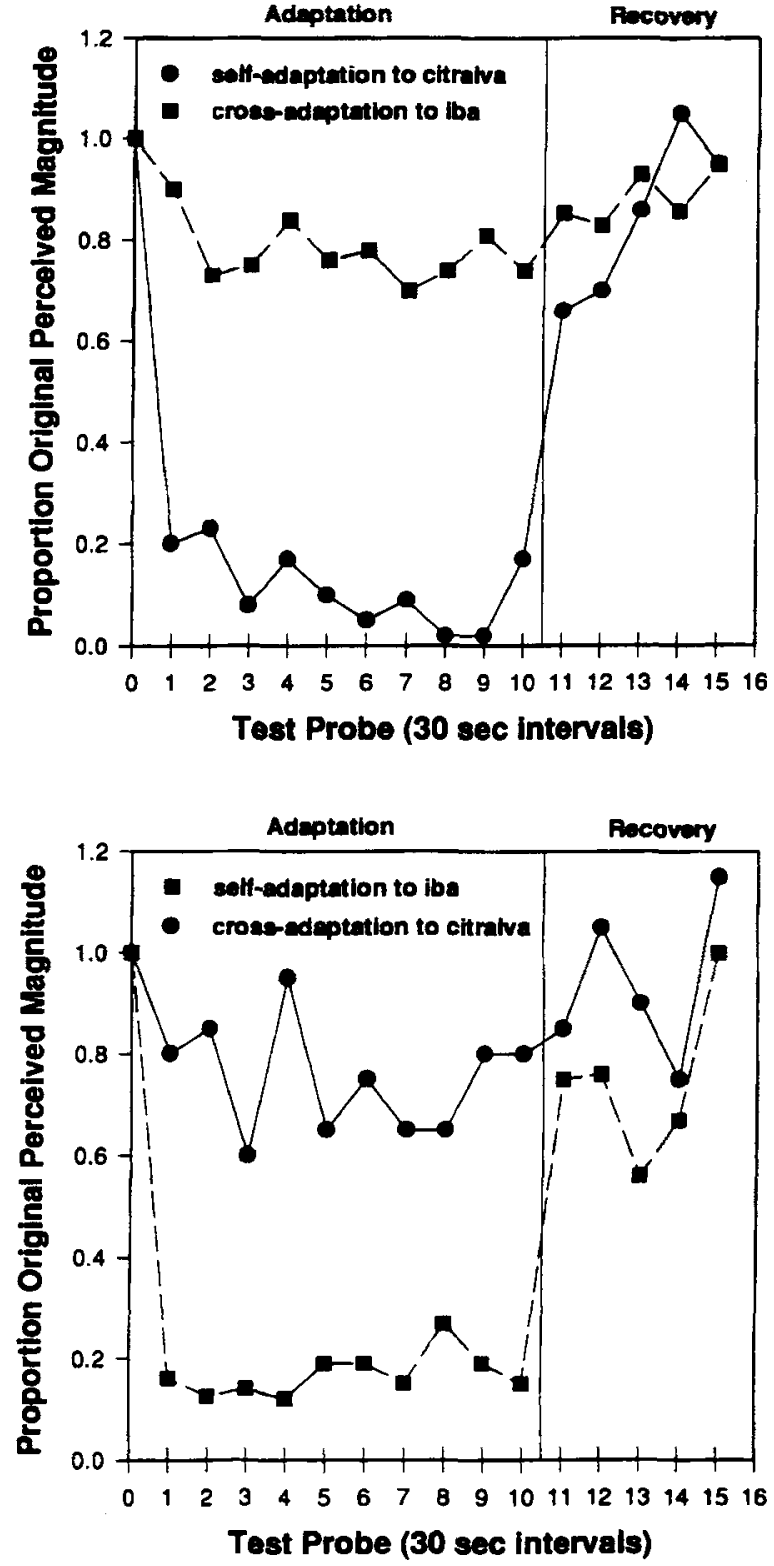

Figure 1. Perceived intensity of iso bornyl acetate and citralva during adaptation to citralva (top panel); perceived intensity of iso bornyl acetate and citralva during adaptation to iso bornyl acetate (bottom panel).

timate for that odor, the differences were not highly significant [for citralva, $F(1,7)=7.42, p=.03$; for iso bornyl acetate, $F(1,7)=5.37, p<.05]$. Repeated measures ANOVAs comparing the self-adaptation ratings with the cross-adaptation ratings for each odorant revealed significant differences for both citralva $[F(1,7)=$ $133.37, p<.05]$ and iso bornyl acetate $[F(1,7)=227.91$, $p<.05]$.

\section{Discussion}

Exposure to both citralva and iso bornyl acetate produced significant decreases in perceptual intensity (selfadaptation) that reached asymptotic levels for all sub- 
jects after only $15 \mathrm{sec}$ of exposure. Importantly, there was no evidence of equivalent cross-adaptation. Although ratings to the control odorant decreased minimally during the adaptation phase, we did not take this as evidence of cross-adaptation. In extensive studies of self- and crossadaptation for numerous odorants, Köster (1971) observed that the act of continuous sniffing of one odorant always produced some suppression in rated intensity of probes of another odorant; he concluded, and we concur, that this suppression is different from odorant-specific crossadaptation.

The self-adaptation did not persist following odorant removal. Intensity estimates given to test probes of each of the adapting odorants showed complete recovery from adaptation within $2.5 \mathrm{~min}$, similar to other short-term olfactory adaptation studies (e.g., Cain, 1974; Steinmetz et al., 1970). The main goal of this investigation, however, was to describe the dynamics of adaptation to odors present for prolonged periods in the natural environment. To what degree does the reduced odor intensity from short-term olfactory adaptation resemble the loss of odor intensity that follows exposures of longer durations? In Experiment 2 we significantly increased the frequency and duration of odor exposure in an attempt to answer that question.

\section{EXPERIMENT 2 \\ Long-Term Exposure, Adaptation, and Recovery}

Anecdotal reports that long-term exposure to an odor reduces or even eliminates subsequent perception of that odor are quite common. If the perceptual changes that follow prolonged odor exposures are the result of odorantspecific olfactory adaptation, like that seen for short exposures, exposed individuals should exhibit elevated detection thresholds and/or a reduction in suprathreshold intensity ratings for the adapting odorant only (see, e.g., Engen, 1982; Pryor, Steinmetz, \& Stone, 1970; Steinmetz et al., 1970). However, if the perceptual changes are produced by the redirection of attentional resources from a familiar stimulus, threshold or suprathreshold measurement tasks (which, by their nature, direct attention to the stimulus) should be relatively unaffected.

We were particularly interested in determining whether the exposure effects on psychophysical measures performed in the laboratory were mirrored by changes in odor perception in the exposure environment. Frequently, individuals who become adapted to a persistent odor in the natural environment experience reduced odor intensity while in the presence of that odor. However, they are rarely tested in order to document a change in olfactory sensitivity, Deficits in olfactory sensitivity as a correlate or consequence of chronic odor exposure have been assessed primarily in chemical-exposed worker populations or in investigations of the effects of pollutants on olfactory perception (e.g., Ahlström et al., 1986; Schwartz et al., 1991). Because these studies measured olfactory sensitivity following exposure, however, they cannot doc- ument the degree or time course of the change in olfactory sensitivity that exposure to an odorant can produce within an individual. ${ }^{4}$

Experiment 2 was designed to (1) obtain baseline measures of sensitivity and intensity, (2) expose subjects to an odorant for 2 weeks and test them at weekly intervals to assess the development of adaptation, and (3) continue to test subjects following odorant removal to record recovery from adaptation. A combined field and laboratory investigation was used primarily because it was impractical to expose individuals to odorants in the laboratory for the frequency and duration necessary to simulate real-world exposure. Moreover, it seemed desirable to develop an exposure procedure in which subjects would experience odors in an ecologically valid way. However, merely assessing changes in the perceived intensity of the odor in the home could overestimate the degree of sensory adaptation, particularly if attentional processes were involved in adaptation. The procedure we employed required 2 weeks of exposure in the home coupled with weekly psychophysical assessments of the individual's response to that odor.

Short-term adaptation is usually measured either as a threshold change (see, e.g., Berglund et al., 1971; Cheesman \& Mayne, 1953) or as a change in perceived intensity of suprathreshold concentrations (see, e.g., Cain, 1970). Although threshold shifts are often used to predict shifts at suprathreshold levels, these methods are not necessarily interchangeable. Therefore, as recommended by Steinmetz et al. (1970), we used both methods to index changes indicative of adaptation. Comparisons of these various measures could yield a more comprehensive picture of the time course, specificity, and conditions underlying long-term olfactory adaptation. Additionally, by measuring thresholds and suprathreshold intensity to concentrations of both the exposure and a control odorant, we could confirm or refute the heretofore anecdotal claim that prolonged exposure to an odorant produces a durable change in sensitivity or perceived intensity to the adapting odorant that can persist for days beyond exposure.

\section{Method}

\section{Subjects}

Eight females were recruited from the University of Pennsylvania community. Their ages ranged from 18 to 42 years, with a mean of 23.75 years. All subjects were screened for the absence of active head cold or allergy, ability to perform a threshold and scaling task, and sensitivity to the odorants used in the experiment. They were paid for their participation. None of the subjects had participated in Experiment 1.

\section{Test Stimuli}

Threshold stimuli. Odorants were diluted in odorless, light, white mineral oil and presented in $270-\mathrm{ml}$ polypropylene squeeze bottles with plastic flip-top caps. Each bottle contained $10 \mathrm{ml}$ of the diluted odorant. Blanks consisted of $10 \mathrm{ml}$ of mineral oil without odorant. A 26-step binary dilution series was prepared for iso bornyl acetate and citralva. The dilution scheme for iso bornyl acetate $(\mathrm{MW}=196.3$ ) ranged from $5.01 \mathrm{mM}$ (Step 0: $1 \times$ $10^{-1 \%} / \mathrm{v} / \mathrm{v} ; 985 \mathrm{mg} / \mathrm{L}$ ) to $1.36 \mu \mathrm{M}\left(\right.$ Step $25: 3 \times 10^{-8 \%} \% \mathrm{v} / \mathrm{v}$; 
$0.295 \mathrm{mg} / \mathrm{L}$ ). The dilution scheme for citralva $(\mathrm{MW}=149.2)$

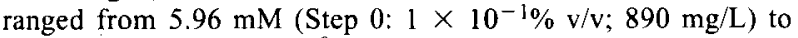
$1.98 \mu \mathrm{M}$ (Step 0: $3 \times 10^{-8 \%} \mathrm{v} / \mathrm{v} ; 0.267 \mathrm{mg} / \mathrm{L}$ ).

Suprathreshold stimuli. The regular suprathreshold stimuli consisted of a series of five concentrations each of citralva and iso bornyl acetate, diluted in odorless, light, white mineral oil and presented in squeeze bottles. Each bottle contained $10 \mathrm{ml}$ of the diluted odorant. For citralva (MW $=149.2)$ the concentrations ranged from $5.96 \mathrm{M}$ (Step 0: $100 \% ; 890 \mathrm{~g} / \mathrm{L}$ ) to $368.63 \mathrm{mM}$ (Step 4: 6/25\% v/v; $55 \mathrm{~g} / \mathrm{L}$ ) in a binary dilution scheme. For iso bornyl acetate the concentrations ranged from 5.01 M (Step 0: $100 \% ; 985 \mathrm{~g} / \mathrm{L}$ ) to $313.3 \mathrm{mM}$ (Step $4: 6.25 \% ; 61.5 \mathrm{~g} / \mathrm{L}$ ) in a binary dilution scheme. The blank contained $10 \mathrm{ml}$ of mineral oil. On the second baseline and the final recovery test, subjects were also tested with a third odorant, amyl acetate, as a control for changes in scale usage. The five concentrations of amyl acetate $(\mathrm{MW}=$ 130.2) ranged from $1.34 \mathrm{M}$ (Step $0: 20 \% \mathrm{v} / \mathrm{v} ; 175 \mathrm{~g} / \mathrm{L}$ ) to $83.71 \mathrm{mM}$ (Step 4: $1.25 \% \mathrm{v} / \mathrm{v} ; 10.9 \mathrm{~g} / \mathrm{L}$ ) in a binary dilution scheme.

\section{Exposure Stimuli}

During the exposure phase, half the subjects were exposed to citralva; the other half were exposed to iso bornyl acetate. The exposure stimulus was constructed in the following manner: A fragrance cartridge filled with absorbent material and covered with a membrane to control evaporation rate (supplied by the Waterbury Co., Waterbury, CT) was filled with $28 \mathrm{~g}$ of a solution containing the odorant in light, white, mineral oil. The fragrance cartridges were supplied with plastic, battery-powered fan-driven fragrance dispensers into which the odorant cartridge was placed (World Wind Dispensers, Waterbury Co., Waterbury, CT). These dispensers continuously odorized the environment. Tests performed by the manufacturer indicated that although loss of odorant would occur at an approximate rate of $5 \mathrm{~g}$ in 2 weeks, the cartridge contained sufficient odorant that the fragrance would remain at the same perceptual intensity in rooms equal to or smaller than $1,500 \mathrm{cu} f \mathrm{ft}$ for at least 3 weeks (Waterbury Co., Waterbury, CT). ${ }^{5}$ The rooms in which the odorant dispensers were placed were all determined to be smaller than 1,440 cu ft.

The concentration of citralva in the odorant cartridge was $20 \%$; the concentration of iso bornyl acetate was $50 \%$. These concentrations were chosen on the basis of the results of a preliminary experiment: Ten subjects ( 6 males, 4 females) sniffed a binary dilution series of iso bornyl acetate ranging from $100 \%$ to $6.25 \%$ and rated the intensity of each stimulus using a labeled magnitude scale (LMS; Green, Shaffer, \& Gilmore, 1993). The 50\% solution of iso bornyl acetate was chosen because it was consistently rated in the moderately strong range on the LMS. Subjects then matched the intensities of citralva to the $50 \%$ iso bornyl acetate solution; $20 \%$ citralva matched the $50 \%$ iso bornyl acetate solution.

\section{Procedure}

Instructions to subjects. Subjects were required to return at the same time and on the same day each week for testing in the laboratory. However, the test time differed for each subject to facilitate work/study schedules; some subjects were tested shortly after leaving the exposure environment in the morning and others were tested after spending as many as $7 \mathrm{~h}$ away from their home environment. They were asked to maintain a constant dietary and activity routine on the day prior to testing. Subjects were not restricted in their use of fragrance, with the exception that they were asked to refrain from using any new fragrances or odorized products for the entire duration of the study.

Schedule of tests. Table 1 shows the schedule of testing and exposure for all subjects. All subjects were tested at weekly intervals for 5 weeks. The first two tests (B1 and B2), prior to home exposure, took place on the 1st and 8th days of the subjects' participation. On the 8th day, the subjects were given the fragrance dispenser and commenced exposure. Subjects were instructed to place the

\begin{tabular}{|c|c|c|}
\hline \multicolumn{3}{|c|}{$\begin{array}{c}\text { Table 1 } \\
\text { Protocol for Long-Term Exposure } \\
\end{array}$} \\
\hline Test Session & Day & Exposure Condition \\
\hline $\begin{array}{l}\text { B1 Baseline Test } 1 \\
\text { (no exposure) }\end{array}$ & 1 & $\begin{array}{l}\text { Preexposure laboratory test to } \\
\text { measure baseline sensitivity }\end{array}$ \\
\hline $\begin{array}{l}\text { B2 Baseline Test } 2 \\
\text { (no exposure) }\end{array}$ & 8 & $\begin{array}{l}\text { Preexposure laboratory test } \\
\text { Immediately following, subjects } \\
\text { begin exposure at home }\end{array}$ \\
\hline Al Adaptation Test 1 & 15 & Home exposure to odorant \\
\hline
\end{tabular}

(1 week of exposure)

A2 Adaptation Test 2

( 2 weeks of exposure)

IH In-Home Test

(24-h recovery)

R1 Recovery Test 1

(1 week of recovery)

R2 Recovery Test 2

( 2 weeks of recovery)

22 Subjects discontinue odorant exposure at home

23 Subjects tested in exposure environment with odor removed

29 Odor removed for 1 week

35 Odor removed for 2 weeks

fragrance dispenser in their dormitory room/bedroom in a central location (but not in front of a window), where it was to run continuously for 2 weeks. During the course of the study, subjects were asked to keep the window(s) closed as much as possible. Because testing took place during the late winter/early spring, compliance with this requirement was not reported to be a problem.

Subjects were tested twice during the exposure phase, on Day 15 (Test A1) and on Day 23 (Test A2). Prior to coming to the laboratory on Day 23, subjects removed the battery from the fragrance dispenser, sealed the unit in a special plastic bag, and returned it to the laboratory. Twenty-four hours following the removal of the fragrance from the environment, the subjects were tested in their home/ dormitory room. Two additional tests were administered during the recovery phase, on Day 29 (Test R1) and Day 36 (Test R2).

Threshold detection. Olfactory detection thresholds were obtained for each of the two odorants, citralva and iso bornyl acetate, using a two-alternative forced choice, up/down, staircase method with a five reversal criterion. On each trial, subjects were presented with a bottle containing the stimulus and a blank (in random order), and after sniffing from each, sequentially, were asked to identify the bottle containing the odorant. An incorrect detection on any trial resulted in the presentation of the next higher concentration, while two consecutive correct detections at a given concentration resulted in the presentation of the next lower concentration. If, at any time, a subject moved more than three consecutive steps either up or down, all reversals up to that point were disregarded and four more reversals were required. In order to further control for thresholds that appeared to "drift" up or down, an extra criterion was added: To be counted, the final reversal (hit or miss) had to occur at a concentration where another corresponding reversal (hit or miss) occurred during the last four. If the subject achieved five reversals but did not meet the additional criteria, testing was continued until the criteria were met. The threshold was calculated from the mean of the last four reversals. Testing could be terminated under four different conditions: (1) Five valid reversals were achieved and the extra criteria were met, resulting in a threshold value; (2) a subject failed to correctly detect the stimulus at the highest concentration; (3) the subject correctly identified the stimulus on two consecutive trials at the lowest concentration; (4) the subject did not meet the criteria for a threshold before the 40 th trial. Condition 4 did not result in a usable threshold value. Condition 2 was considered evidence of a subject's complete inability to detect the stimulus in the test context.

Thresholds were obtained for both odorants in the same test; that is, citralva and iso bornyl acetate were presented on alternating trials until the threshold criteria were reached for each odorant separately. 
Table 2

Mean Detection Thresholds for Control and Adapting Odorant at Baseline (B1, B2), Adaptation (A1, A2), Recovery (R1, R2) and Test In Home (IH)

\begin{tabular}{|c|c|c|c|c|c|c|c|c|c|c|c|c|c|c|}
\hline \multirow[b]{2}{*}{ Odorant } & \multicolumn{2}{|c|}{ B1 } & \multicolumn{2}{|c|}{$\mathrm{B} 2$} & \multicolumn{2}{|c|}{ Al } & \multicolumn{2}{|c|}{ A2 } & \multicolumn{2}{|c|}{$\mathrm{R} 1$} & \multicolumn{2}{|c|}{$\mathrm{R} 2$} & \multicolumn{2}{|c|}{$\mathrm{IH}$} \\
\hline & $M$ & $S D$ & $M$ & $S D$ & $M$ & $S D$ & $M$ & $S D$ & $M$ & $S D$ & $M$ & $S D$ & $M$ & $S D$ \\
\hline Control & 7.91 & .85 & 11.47 & 1.50 & 12.03 & 1.70 & 13.91 & 1.80 & 14.28 & 1.90 & 15.41 & 1.80 & 15.46 & 2.10 \\
\hline Adapting & 8.11 & 1.01 & 12.06 & 1.40 & $9.94^{*}$ & 1.05 & $9.31^{*}$ & .96 & $10.34^{*}$ & 1.78 & $12.34^{*}$ & 1.98 & $9.46^{*}$ & .74 \\
\hline
\end{tabular}

*Significantly different from control odorant $(p<.01)$.

In all cases, we used the threshold value representing the first threshold obtained for a given odorant, despite the fact that continued presentation occasionally resulted in an apparent shift in threshold.

The starting concentration used for both odorants on the first baseline test was Step 6. On subsequent tests, the starting concentration used was one step weaker than the threshold obtained on the previous test.

Suprathreshold intensity estimates. In addition to obtaining detection thresholds, subjects were asked to rate a series of suprathreshold stimuli for intensity using a labeled magnitude scale (LMS; Green, Shaffer, \& Gilmore, 1993). The LMS is a categoryratio scale that allows subjects to scale and rate the intensity of stimuli using natural language descriptors. With this scale, ratings of odor intensity are made in the context of all previous experience with odors. The scale has been validated, against magnitude estimation, for olfactory stimuli; ratings obtained with this scale have ratio properties equivalent to magnitude estimation (Green et al., in press).

The suprathreshold intensity judgments always followed the threshold test, on the same schedule (Table 1). The test series consisted of the five concentrations each of citralva and iso bornyl acetate described earlier plus one blank. Five concentrations of a third odorant, amyl acetate, were added to the suprathreshold test series on two occasions: at the second baseline test (B2) and again at the second recovery test (R2). Thus, each subject always rated the perceived intensity of a control odor (to which he/she was exposed only at test) as well as the adapting odor; on two occasions they rated an odor that appeared only in that test. The ratings of perceived intensity to the control odor (either iso bornyl acetate or citralva) were obtained as a control for general changes in perceived intensity as a function of adaptation to a single odorant. The ratings of intensity to the amyl acetate were collected to control for changes in scale usage over the weeks of the study. At test, subjects were presented with each bottle twice in an individually randomized sequence for a total of 22 judgments, except at Test B2 and R2, in which the inclusion of amyl acetate made for a total of 32 judgments.

Questionnaires. During the exposure phase subjects also were given questionnaires that asked for the following information: (1) the number of hours spent each day in the odorized environment, (2) quality descriptions of the odorant, (3) intensity estimates of the odorant in the environment, (4) the duration of odor perception upon returning to their room, and (5) comments made by visitors to their room who noticed the odor.

Each questionnaire was to be filled out no more than $24 \mathrm{~h}$ prior to their third and fourth test (which took place following 1 and 2 weeks of exposure, respectively) and returned during the next appointment. At each appointment subjects were also asked to indicate the number of hours that had elapsed since their last exposure to the odorant.

\section{Results}

\section{Threshold Detection}

All thresholds were expressed as binary dilution steps of the odorant series, with Step 0 as the strongest concentration and Step 25 as the weakest. Thus, increasing numbers represented greater sensitivity and decreasing numbers represented less sensitivity. The individual thresholds obtained at each test were averaged across subjects for the control odorant and the adapting odorant separately. Again, for 4 subjects, the control odorant was iso bornyl acetate and the adapting odorant was citralva; the reverse was true for the remaining 4 subjects.

An omnibus ANOVA with type of odor (control vs. adapting) and test session (B1 or B2, A1 or A2, R1 or $\mathrm{R} 2)$ as within-subject variables was performed on the thresholds. Thresholds varied significantly across tests $\left[F(6,42)=5.923, M S_{\mathrm{e}}=8.8, p<.01\right]$. Of greatest importance, however, was the finding that there was a significant interaction of type of odor (control vs. adapting) and test session $\left[F(6,42)=3.53, M S_{\mathrm{e}}=6.75, p<.01\right]$. Post hoc comparisons on the means, which are summarized in Tables 2 and 3, provided a more complete description of shifts in detection thresholds across the weekly test sessions.

1. Baseline. Table 2 shows that baseline thresholds for the control and the adapting odorant did not differ at either Test 1 or 2 . However, thresholds shifted significantly between Tests 1 and 2 for both the control and the adapting odorants. Figure 2, which depicts the average thresholds for the 8 subjects across all six laboratory tests (and the one in-home test), shows this difference most clearly. The $y$-axis depicts thresholds in binary dilution steps, where 0 is the highest concentration and 25 is the weakest. The $x$-axis depicts the six test sessions (which are separated by 1 week). Thresholds showed approximately a 16-fold shift between these two baseline tests, indicating a significant increase in sensitivity to both odorants.

2. Adaptation. We saw reliable evidence of the development of adaptation during this period as sensitivity to the adapting odorant decreased steadily from the final baseline test to the two tests during the exposure phase. ${ }^{6}$ By the end of 1 week of exposure, 6 of the 8 subjects had shown a measurable, albeit not significant, loss of sensitivity. Following 2 weeks of exposure, the loss of sensitivity was significant. Six of the 8 subjects showed losses in

Table 3

Summary of Significance Tests Comparing Threshold at Final Baseline (B2) With Thresholds at Other Test Sessions

\begin{tabular}{lccccccc}
\hline Odorant & B1 & B2 & A1 & A2 & R1 & R2 & IH \\
\hline Control & $*$ & - & n.s. & n.s. & $*$ & $*$ & $*$ \\
Adapting & $*$ & - & n.s. & $*$ & $*$ & n.s. & $*$ \\
\hline
\end{tabular}

Note-B1, baseline; A1, A2, adaptation; R1, R2, recovery; IH, test in home. *Significantly different from threshold at B2; n.s., not significantly different from threshold at B2; -, planned comparison not made. 


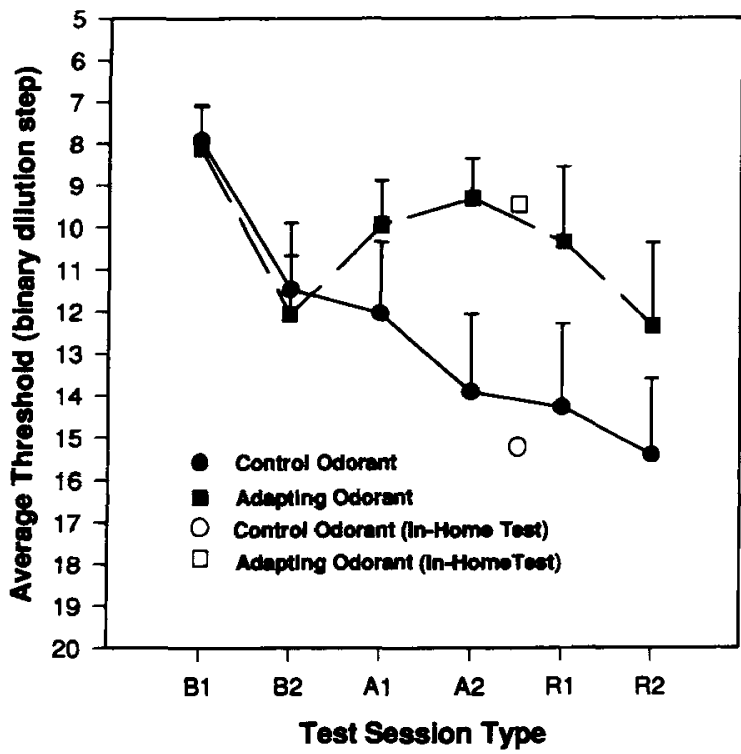

Figure 2. Average olfactory detection thresholds for the control and adapting odorant across the seven test sessions (two baseline, adaptation, and recovery tests, one in-home test).

sensitivity at the end of the first week and all showed losses by the final test during exposure. In contrast, their apparent sensitivity to the control odorant increased across tests in the exposure phase, although not significantly. As Table 2 shows, there was a significant difference between thresholds for control and adapting odorants following 2 weeks of exposure to the adapting odorant.

3. Recovery. Following removal of the odorant from the bedroom, we saw sensitivity to the adapting odorant increase. The last threshold obtained during recovery did not differ from the threshold obtained during the final baseline test for the adapting odorant. However, sensitivity to the control odorant continued to increase during the recovery phase. Both thresholds obtained during the recovery phase for the control odorant were significantly different from that at baseline. ${ }^{7}$ Thresholds for the control and adapting odorant were still significantly different at the final test.

4. Home test (recovery). Twenty-four hours following odorant removal, our standard assessment of olfactory perception (detection threshold and perceived intensity ratings) was conducted in the subject's exposure room. As shown in Table 2, detection thresholds for the control and the adapting odorant were significantly different. However, a comparison with the thresholds obtained in the laboratory $24 \mathrm{~h}$ earlier revealed no significant differences for thresholds obtained in the two contexts.

\section{Suprathreshold Intensity Estimates}

To analyze the intensity ratings for the suprathreshold odors, all scale values (which ranged from 0 to 95 units) were converted to log values. This was done because data collected with this scale previously were logarithmically distributed (Green et al., 1993); these data were no exception. The ratings made during the in-home tests were recorded on paper rather than with the computer used in the laboratory, so the home data were rescaled from the paper scale to the computer scale. For each test, ratings were averaged across replicates at each concentration.

Amyl acetate was included at the second baseline and the final recovery test as an additional control for changes in scale usage. The intensity ratings for amyl acetate were unchanged across the two tests. Analyses revealed no main effect of test or a significant interaction of test with concentration $(F \mathrm{~s}<1)$. Because there was no apparent change in scale usage for an unadapted odorant that was tested only twice, we subsequently disregarded these data.

In general, ratings of the suprathreshold stimuli across the weekly tests did not shift as much as the detection thresholds did. We first compared the ratings given to the control and adapting stimuli (and the blanks) only within the two tests in each phase (B1 vs. B2, A1 vs. A2, R1 vs. $\mathrm{R} 2$ ). Perceived intensity ratings did not significantly differ $(F \mathrm{~s}<1)$. Thus, for presentation simplicity, we collapsed the ratings across the two baseline tests, the two adaptation tests, and the two recovery tests. Combined with the in-home test, this yielded four separate tests of 11 ratings ( 5 for the control odor, 5 for the adapting odor, 1 for the blank) for each subject.

A repeated measures ANOVA of the intensity ratings of the control odorant revealed an expected main effect of stimulus concentration $\left[F(4,28)=16.37, M S_{\mathrm{e}}=.016\right.$, $p<.01]$ and a significant interaction between test and concentration $\left[F(12,84)=2.287, M S_{\mathrm{e}}=.011, p<.01\right]$. The top panel of Figure 3 illustrates the reason for the interaction. Subjects did not appear to be able to discriminate well among concentrations of the control odorant at the in-home test.

The same analysis of intensity ratings of the adapting odor revealed both a significant main effect of stimulus concentration $\left[F(4,28)=8.635, M S_{\mathrm{e}}=.036, p<.01\right]$ and test $\left[F(12,84)=3.635, M S_{\mathrm{e}}=.098, p<.01\right]$. No significant interaction was present. Post hoc tests on the mean ratings for the different concentrations revealed significant differences in intensity ratings between the baseline and adapting phases only. However, as shown in the bottom panel of Figure 3, the reduction in perceived intensity was exhibited only for the two weakest concentrations of the series.

A repeated measures ANOVA on the ratings for the blank stimuli showed a main effect of test phase $[F(3,7)=$ $\left.5.82, M S_{\mathrm{e}}=.246, p<.01\right]$. Post hoc comparisons isolated the group that differed significantly from the others: Ratings given to the blanks during the adaptation phase were significantly lower than ratings to the same stimuli during baseline, recovery, or in-home test.

\section{Questionnaires: Correlation of Field and Laboratory Measures}

All subjects recorded the number of hours per day spent in the presence of the odorant. Analysis of these records revealed a significant correlation between the number of hours spent in the presence of the odorant and the onset of 


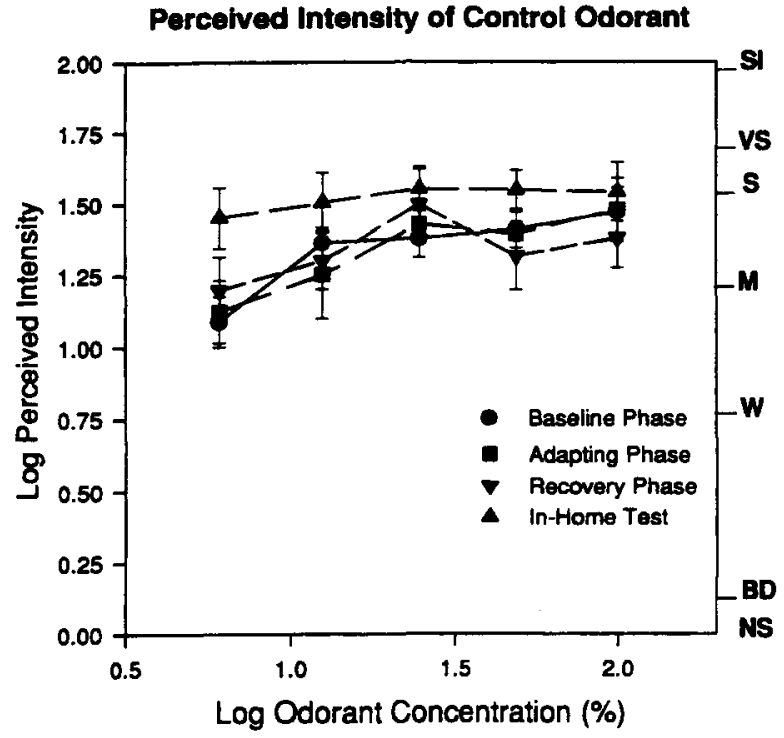

Perceived Intensity of Adapting Odorant

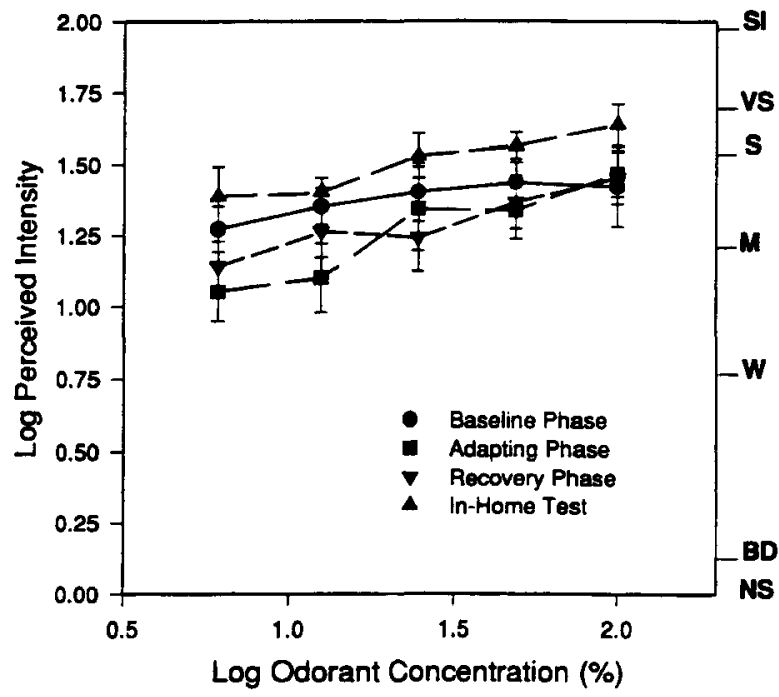

Figure 3. Changes in the perceived intensity of the five concentrations of the control (top panel) and the adapting (bottom panel) odorant during baseline, adaptation, recovery phase, and in-home test. SI, strongest imaginable; VS, very strong; S, strong; $M$, moderate; W, weak; BD, barely detectable; NS, no sensation

adaptation as measured in the laboratory. The 5 subjects who reported having $>70 \mathrm{~h}$ per week of odorant exposure showed significant adaptation following 1 week of exposure $\left(r^{2}=.74\right)$. The degree of adaptation for those subjects appeared to reach an asymptote in the first week. Subjects with less than $70 \mathrm{~h}$ of exposure in Week 1 did not show significant adaptation until 2 weeks of exposure.

Interestingly, subjects who were tested within 1 to $2 \mathrm{~h}$ of the most recent exposure showed no difference in the amount or degree of adaptation compared with those subjects who were tested more than 4 to $7 \mathrm{~h}$ after exposure $\left(n=8, r^{2}=.09\right)$. It is conceivable that the 1 -h delay between exposure and test (experienced by all sub- jects) may have been sufficient to eliminate any residual short-term adaptation that was present during and immediately after exposure. Alternatively, because of the range of normal variability in individual thresholds, it could be argued that threshold measures were insensitive to any between-subjects temporal variation.

Subjects chose from the following list to describe the quality of the odorant in their home: musky, medicinal, heavy, light, warm, cold, citrusy, pine oil, disinfectant, woody, fruity, spicy, floral, sweet, herbal, perfumery, camphor, incense, soapy, and lemony. Although descriptor profiles showed some individual variability, there was also strong agreement on quality descriptors for the two odors: Citralva was most often described as citrusy, fruity, light, and warm; iso bornyl acetate was most often described as piney, woody, medicinal, disinfectant, and heavy. These descriptions are consistent with previously published data on the character profiles of these two odorants (Dravnieks, 1985). There were no systematic changes in subjects' descriptions of the odorant from Week 1 to 2 of the exposure phase.

Subjects were also asked to rate the intensity of the odor in their home. These data were collected at the beginning of the exposure phase and following 1 and 2 weeks of exposure. The intensity ratings at Week 1 and Week 2 were converted to proportions of the initial rating. Figure 4 shows the scatterplot of the subjects' intensity ratings and their corresponding detection thresholds to the adapting odorant for each of the 2 weeks of exposure. Significant correlations were observed $\left(r^{2}=.72\right.$ for citralva; $r^{2}=.80$ for iso bornyl acetate; $p$ s $<.05$ ) between the subjects' rating of odor intensity in their home environment and their loss of sensitivity to that odor.

One method that has been used to index short-term adaptation is to obtain ratings of odor intensity during continuous or intermittent exposure (see, e.g., Cain, 1970, 1974). With this method, continued exposure produces a rapid reduction in the perceived odor intensity, as in Experiment 1 , where self-adaptation to these substances reached asymptotic levels within $15 \mathrm{sec}$. Although this method was not used to measure adaptation in Experiment 2 , data from the questionnaire may have some bearing on identifying the short-term adaptation that may occur during repeated, long-term exposures. For each day of the exposure phase, subjects were asked to estimate how long they continued to smell the odor upon entering the room. During the first week, all individuals reported being able to smell the odorant for up to $1 \mathrm{~h}$ after entry. By the second week of exposure, however, a few subjects reported smelling the odor for only about $15 \mathrm{~min}$ after entering the room; the majority reported smelling it for even briefer durations (e.g., 1-2 min). As verification that the odorant was still perceptible, all subjects did report that unexposed visitors to their home or dorm room during both weeks of exposure perceived the odor readily.

At the final test, we asked the subjects to report what they believed would be the outcome of exposure to this odorant (e.g., would living with the fragrance in their home affect their ability to smell it?). Interestingly, all 


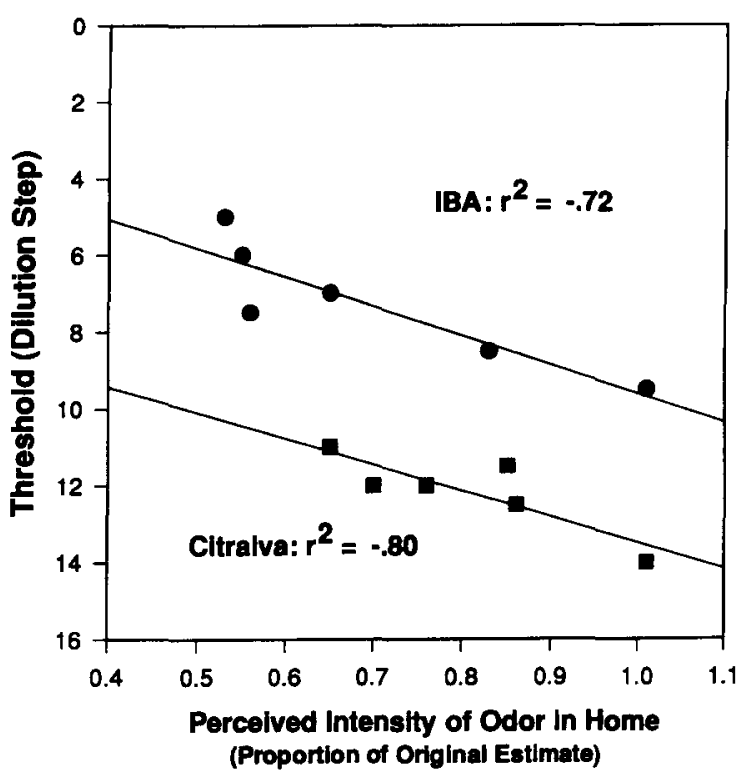

Figure 4. The relationship between loss of perceived intensity in the home and sensitivity as measured in the laboratory. Average detection thresholds for citralva and iso bornyl acetate (IBA) are plotted separately.

but 1 subject said they expected that exposure to the odorant would make them more sensitive and they would be able to perceive it more readily.

\section{Discussion}

In Experiment 2 we combined both field (in-home) exposures and laboratory assessments to explore the effects of long-term exposure to an odorant on the perceptual response (both at threshold and above threshold) to that odorant. Individuals who were exposed for a prolonged period to a pleasant odor in their home showed a marked and significant decrease in their ability to detect that odorant. In as little as 1 week, this exposure produced an elevation in detection thresholds that was measurable in the laboratory, in some cases more than $6 \mathrm{~h}$ after being exposed. The elevation in threshold persisted for days and for some individuals for more than a week following removal of the odorant from their home.

Whereas long-term exposure in the home produced a loss of sensitivity to the adapting odorant, intermittent exposure in the laboratory produced increased sensitivity to the control odorant. Across the six laboratory tests, thresholds to the control odor decreased an average of eight binary dilution steps or 256-fold. Increased sensitivity to an odor following repeated threshold tests for that odor is consistent with previous reports (e.g., Rabin \& Cain, 1986; Semb, 1968), does not appear to involve odorant-specific receptor sensitization, and may represent practice effects on the task as well as perceptual learning in the detection of the odorant(s).

However, increased sensitivity to the control odorant is of special significance for measuring the degree of adaptation in this study. If we index adaptation using only the loss of sensitivity to the adapting odorant, we may seriously underestimate the amount of olfactory adaptation. From the first to the second test (prior to any exposure), detection thresholds decreased significantly for both the control and the adapting odors. Therefore, in the absence of any exposure manipulation, repeated testing of the adapting odorant should increase sensitivity as it did for the control odorant. Because it does not increase, but in fact, significantly decreases, we suggest that the obtained threshold for the adapting odor is the product of two competing processes: adaptation and sensitization. If the sensitization from experience at test masks the actual amount of adaptation, the actual degree of adaptation is best estimated by assessing the net difference between the loss of sensitivity to the adapting odorant and the gain in sensitivity by the control odorant.

The effects of adaptation from long-term exposure were more salient at threshold levels than at suprathreshold levels. Exposure produced a small but significant decrease in the perceived intensity of the adapting odorant at the weakest suprathreshold concentrations, whereas ratings of the control odorant did not change. The lack of comparable adaptation effects at threshold and suprathreshold concentrations may well be a function of the concentration range we employed. Whereas the concentration range for threshold tests was fixed by the individual's threshold, the range for suprathreshold tests was an arbitrary choice. It is conceivable that suprathreshold stimuli spanning a weaker concentration range would have shown greater effects of adaptation. However, finding adaptation effects restricted to the weakest concentrations is consistent with previous evidence showing that adaptation does not change the intensity ratings for concentrations higher than the adapting stimulus (see, e.g., Todrank et al., 1991) and that occupational exposure to odorants reduces the perception of weak concentrations of the odorant, while stronger concentrations are relatively unaffected (i.e., "loudness recruitment"; Ahlström et al., 1986).

It is also interesting that intensity ratings of the blank stimuli in the suprathreshold tests changed systematically across the test phases. Although the present research cannot determine the cause of this effect, consistently lower ratings to the blanks during the adaptation phase could indicate either a criterion shift in the perceived odor intensity of "noise" stimuli (e.g., Green \& Swets, 1966) or a true shift in the sensitivity to the background. The role that decision processes play in the perception of odors is likely to be quite substantial and needs further exploration.

\section{GENERAL DISCUSSION}

In the present research we investigated the development of olfactory adaptation across two different time periods. In Experiment 1, we measured short-term olfactory self- and cross-adaptation to two compounds and showed, consistent with many other studies, that significant reduction in odor intensity can be achieved with as 
little as $15 \mathrm{sec}$ of prolonged sniffing. In Experiment 2 we extended the duration and frequency of exposure to an odorant and demonstrated that long-term naturalistic exposure to an odorant can produce self-adaptation on threshold and suprathreshold stimuli. To the extent that we accept the forced-choice threshold task as a bias-free measure of olfactory sensitivity, the outcome of Experiment 2 provides evidence that the reductions in odor intensity from prolonged exposure in natural environments are due, in part, to sensory adaptation, not simply to attentional mechanisms. We now consider the degree to which this long-term adaptation reflects the same processes as do short-term adaptation.

\section{Short-Term Versus Long-Term Adaptation}

At the outset of this paper, we raised the question of whether the adaptation produced by repeated, prolonged odorant exposure would differ qualitatively or just quantitatively from short-term olfactory adaptation. Following 1 week of a minimum of $6 \mathrm{~h}$ daily exposure, 6 of the 8 subjects exhibited reduced sensitivity to the adapting odorant; by the end of 2 weeks this was true for all subjects. But can we infer that the underlying process differs from that producing short-term adaptation? Because subjects spent at least $6 \mathrm{~h}$ in the presence of the odorant during the night prior to their test, it could be argued that the subsequent shift in detection threshold seen in the laboratory simply resulted from a daily renewal of short-term adaptation, with perhaps more rapid readaptation upon subsequent exposures (e.g., savings in adaptation, Thompson \& Spencer, 1966). In Experiment 2, the subjects reported that daily adaptation to the odor proceeded more rapidly as the exposure phase progressed, suggesting that an important feature of the development of long-term adaptation may involve some savings. The locus of a savings mechanism could be peripheral, resulting from more rapid response by the receptors; it could be central, resulting from more rapid activation of the memory representation of the odor; or it could occur at both stages, with feedback between these two endpoints. As evidence of the potential complexity of mechanisms of olfactory adaptation, one can turn to studies using ipsalateral and contralateral odorant delivery to identify the locus of adaptation (e.g., Köster, 1971). Results from these studies suggest the existence of two adaptation processes: a central process (e.g., olfactory bulb or higher) and a peripheral process in which the rates of recovery from adaptation appear to differ. Ultimately, when data from behavioral and cellular studies of olfactory adaptation are merged, it would not be surprising to discover that olfactory adaptation, particularly to odors in natural environments, is a complex, multilocus process.

Alternatively, recent reports have indicated that certain organic chemicals can be found in the blood for many hours or even days after inhalation (Benignus, Muller, Graham, \& Barton, 1984). Because olfactory receptor cells could be stimulated by these absorbed chemicals either when they are expired or if they are directly diffused from capillaries (Maruniak, Silver, \& Moulton,
1983), receptor-mediated short-term adaptation may be occurring continuously throughout the 2 weeks of exposure, not just when subjects are in the presence of the odorant cartridge.

The most significant difference between long-term and short-term olfactory adaptation appears in responses made during recovery. Data collected during the recovery phase of Experiment 2 show that olfactory detection thresholds are slow to recover to preexposure levels. One week following removal of the home odorant 2 subjects showed complete recovery and 2 showed incomplete recovery, but 4 showed no recovery. At the final test, 2 weeks following odorant removal, 4 subjects showed complete or more than complete recovery (thresholds lower than baseline levels), but 3 still had not fully recovered from adaptation and 1 subject had not begun to recover. After 2 weeks with no further exposure to the adapting odor outside of the test sessions, half the subjects had not regained their preexposure levels of sensitivity. In recent work we have replicated this study, increasing the number of recovery tests and extending the recovery period. Preliminary data suggest that it can take up to 4 weeks following odorant removal for the thresholds for the adapting and the control odorants to converge.

It is important to note that during the laboratory assessments subjects never became anosmic to the test odor or lost their ability to detect the odor at any concentration during threshold tests. Yet, at the end of 2 weeks of exposure, many individuals reported that they no longer smelled the odor in their home. This report led us to suspect that testing subjects in their home environment might reveal a further reduction in sensitivity that was context specific. This was not confirmed. In fact, sensitivity to the adapting odorant in the home test was equivalent to sensitivity measured in the laboratory. There are several reasons why self-reports of odor perception and measured threshold levels might differ, however. Thresholds in the home were assessed $24 \mathrm{~h}$ following the removal of the odorant and it is possible that subjects achieved their maximal loss of sensitivity when the odor was present. At the test, which occurred $24 \mathrm{~h}$ following odorant removal, some recovery might have already occurred.

It could also be argued that the decrease in odor intensity in the exposure environment is a combination of two concurrent processes: sensory adaptation, which reduces responsiveness to the detection of an odorant at the level of the specific system; and a decrease in attentional resources allocated to a fixed, familiar signal in the environment (a process often referred to as "habituation"). A psychophysical test of odor perception naturally focuses attention on detection of the odorant. In laboratory studies of olfactory adaptation at suprathreshold levels, it is rarely the case that even with $90 \mathrm{~min}$ of exposure, subjects report the odor has "disappeared." Therefore, attention may have little or no effect on the odor perception that is assessed by psychophysical tasks, although it may be highly influential in terms of the perception of an odor in the exposure context. In that light, it is probably significant that the stimulus odors were relatively pleas- 
ant and familiar to most individuals in their home environment (i.e., scents found in numerous cleaning or airfreshening products). The degree of odor adaptation in natural environments that is attributable to attentional processes may depend on both the pleasantness and the familiarity of the odor (see, e.g., Engen, 1982).

\section{Future Directions}

There are many avenues to pursue to further illuminate the nature of long-term adaptation. In the present experiments we used only one concentration of the adapting odorant. It remains to be determined whether there is a dose-response relationship in the degree of long-term adaptation as there appears to be for short-term olfactory adaptation. In addition, increasing the number of tests during the recovery phase can reveal whether sensitivity to the adapting odorant will recover to the level of the control odorant or even surpass it.

We are also aware that long-term adaptation in natural environments has certain characteristics that were not examined in these experiments. First, it is likely that longterm olfactory adaptation comprises multiple processes: sensory adaptation (indexed by threshold shifts in the present experiments) as well as attentional or memorial processes. Future studies can address the specific contribution of these separate processes to the overall loss of odor perception in real-world environments. In addition, because real-world odor experience is rarely limited to the perception of a single odor (Berglund, 1974; Berglund \& Engen, 1993), future studies should examine longterm adaptation to mixtures.

Finally, there is enormous theoretical and practical importance in understanding the conditions that produce olfactory adaptation. Studies of olfactory learning in other species (e.g., rat, hamster, mouse) have found that longterm exposure to a single, dominant-odor environment has degenerative effects on mitral cells in the olfactory bulb, but no consistent effects on behavioral measures of sensitivity to that odor or other odors (Cunzeman \& Slotnick, 1984; Laing \& Panhuber, 1978, 1980; Panhuber, Mackay-Sim, \& Laing, 1987). Yet some exposure conditions for humans and mice induce odor sensitization (e.g., Wang, Wysocki, \& Gold, 1993; Wysocki, Dorries, \& Beauchamp, 1989). Evidence from other sensory and physiological systems suggests that continuously presented stimuli produce adaptation whereas intermittently presented stimuli do not (for a review, see Post, 1980). These distinctions may prove useful for olfaction as well.

\section{Conclusions}

Exposing individuals for a 2-week period to a pleasant odor in their home environment produced diminished olfactory sensitivity with the following characteristics: The reduction in sensitivity was specific to the adapting odorant and evidenced by elevated thresholds as well as lower intensity ratings to weak concentrations of the odorant. Both of these characteristics are consistent with short-term adaptation effects. However, adaptation in the exposure environment, which was measured by selfreports of odor intensity, appeared more profound and therefore may reflect attentional processes as well as renewal of short-term adaptation (savings) upon repeated exposure.

Some features of long-term adaptation appear to differentiate it from short-term adaptation. The loss of sensitivity was measurable in situations outside the exposure context more than $6 \mathrm{~h}$ after any exposure. Furthermore, this loss was remarkably persistent. Detection thresholds did not return to preexposure levels for most subjects until 2 weeks after removal of the odorant from their home. Comparisons with the control odorant showed an even more persistent effect of adaptation. Because detection of the control odorant improved dramatically over the seven test sessions, thresholds for the adapting odorant were still significantly higher than were thresholds for the control odorant, 2 weeks after odorant removal.

Long-term exposure to an odorant produces a persistent, albeit reversible, decrement in the ability to detect that odorant. Because the olfactory system is required to process signals against a "noisy" background, the filtering of constant unvarying signals that do not convey information is an important function of adaptation (see, e.g., Voight \& Atema, 1990). In this study, we have shown that olfactory adaptation can be more than a transient response and can be observed in a situation other than the environment in which exposure occurs.

\section{REFERENCES}

Ahlström, R., Berglund, B., Berglund, U., Lindvall, T., \& WenNBERG, A. (1986). Impaired odor perception in tank cleaners. Scandinavian Journal of Work \& Environmental Health, 12, 574-581.

Anderson, I., Lundqvist, G. R., Molhave, L., Pederson, O. F., ProcTOR, D. F., VAETH, M., \& WYON, D. P. (1983). Human response to controlled levels of toluene in 6-hour exposures. Scandinavian Journal of Work \& Environmental Health, 9, 405-418.

Benignus, V. A., Muller, K. K., Graham, J. A., \& Barton, C. N. (1984). Toluene levels in blood and brain of rats as a function of toluene level in inspired air. Environmental Research, 33, 39-46.

BERGLUND, B. (1974). Quantitative and qualitative analysis of industrial odors with human observers. In W. S. Cain (Ed.), Odors: Evaluation, utilization and control (Annals of the New York Academy of Sciences, Vol. 237, pp. 35-51). New York: New York Academy of Sciences.

BERGLUND, B. (1977). Quality, intensity and time in olfactory perception (Report No. 505). Stockholm: University of Stockholm, Department of Psychology.

Berglund, B., Berglund, U., Engen, T., \& Lindvall, T. (1971). The effect of adaptation on odor detection. Perception \& Psychophysics, 9, 435-438

BERGLUNd, B., \& ENGEN, T. (1993). A comparison of self-adaptation and cross-adaptation to odorants presented singly and in mixtures. Perception, 22, 103-111.

CaIN, W. S. (1970). Odor intensity after self-adaptation and crossadaptation. Perception \& Psychophysics, 7, 271-275.

CAIN, W. S. (1974). Perception of odor intensity and the time-course of olfactory adaptation. ASHRAE Transactions, 80, 53-75.

CAIN, W. S., \& POLAK, E. H. (1992). Olfactory adaption as an aspect of odor similarity. Chemical Senses, 17, 481-491.

CheEsman, G. H., \& MaYNe, S. (1953). The influence of adaptation on absolute threshold measurements for olfactory stimuli. Quarterly Journal of Experimental Psychology, 5, 22-30.

Cometto-MuÑIZ, J. E., \& CAIN, W. S. (1995). Olfactory adaptation. In 
R. L. Doty (Ed.), Handbook of olfaction and gustation (pp. $257-$ 281). New York: Marcel Dekker.

Cunzeman, P. J., \& Slotnick, B. M. (1984). Prolonged exposure to odors in the rat: Effects on odor detection and mitral cells. Chemical Senses, 9, 351-361.

DRAVNieks, A. (1985). Atlas of odor character profiles. Philadelphia: American Society for Testing and Materials.

Ekblom, A., Flock, A., Hansson, P., \& Ottoson, D. (1984). Ultrastructural and electrophysiological changes in the olfactory epithelium following exposure to organic solvents. Acta Otolaryngologica, 98, 351-361.

Ekman, G., Berglund, B., Berglund, U., \& Lindvall, T. (1967). Perceived intensity of odor as a function of time of adaptation. Scandinavian Journal of Psychology, 8, 177-186.

ENGEN, T. (1982). The perception of odors. New York: Academic Press.

GaGnon, P., Mergler, D., \& LAPARE, S. (1994). Olfactory adaptation, threshold shift and recovery at low levels of exposure to methyl isobutyl ketone (MIBK). Neurotoxicology, 15, 637-642.

GetcheLL, T. V. (1986). Functional properties of vertebrate olfactory receptor neurons. Journal of Physiology, 3, 772-809.

Green, B. G., Dalton, P., Cowart, B., Shaffer, G., Rankin, K., PiERCE, J., \& HigGins, J. (in press). Further evaluations of the utility and "validity" of the labeled magnitude scale. Chemical Senses.

Green, B. G., Shaffer, G. S., \& Gilmore, M. M. (1993). Derivation and evaluation of a semantic scale of oral sensation magnitude with apparent ratio properties. Chemical Senses, 18, 683-702.

GreEN, D. M., \& SwETS, J. A. (1966). Signal detection theory and psychophysics. New York: Wiley.

KöSTER, E. P. (1971). Adaptation and cross-adaptation in olfaction: An experimental study with olfactory stimuli at low levels of intensity. Unpublished doctoral dissertation, University of Utrecht.

LAing, D. G., \& Panhuber, H. (1978). Olfactory sensitivity of rats reared in an odorous or deodorized environment. Physiology \& Behavior, 25, 555-558.

LAING, D. G., \& PANHUBER, H. (1987). Neural and behavioral changes in rats following continuous exposure to an odor. Journal of Comparative Physiology, 124, 259-265.

MARUniaK, J., Silver, W., \& Movlton, D. G. (1983). Olfactory receptors respond to blood-borne odorants. Brain Research, 265, 312-316.

Mergler, D., \& Beauvais, B. (1992). Olfactory threshold shift following controlled 7-hour exposure to toluene and/or xylene. Neurotoxicology, 13, 211-216.

Mozell, M. M. (1962). Olfactory mucosal and neural responses in the frog. American Journal of Physiology, 203, 353-358.

Panhuber, H., Mackay-Sim, A., \& Laing, D. G. (1987). Prolonged odor exposure causes severe cell shrinkage in the rat olfactory bulb. Developmental Brain Research, 31, 307-311.

Pierce, J. D., Wysocki, C. J., \& Aronov, E. V. (1993). Mutual crossadaptation of the volatile steroid androstenone and a non-steroid perceptual analog. Chemical Senses, 18, 245-256.

Post, R. M. (1980). Minireview: Intermittent versus continuous stimulation: Effect of time interval on the development of sensitization or tolerance. Life Sciences, 26, 1275-1282.

POTTER, H., \& CHOROVER, S. (1976). Response plasticity in hamster olfactory bulb: Peripheral and central processes. Brain Research, 116, 417-429.

Pryor, G. T., Steinmetz, G., \& Stone, H. (1970). Changes in absolute detection threshold and in subjective intensity of suprathreshold stimuli during olfactory adaptation and recovery. Perception \& Psychophysics, 8, 331-335.

RABIN, M. D., \& CAIN, W. S. (1986). Determinants of measured olfactory sensitivity. Perception \& Psychophysics, 39, 281-286.

SchieT, F. T., \& CaIN, W. S. (1990). Odor intensity of mixed and unmixed stimuli under environmentally realistic conditions. Perception, 19, 123-132.

Schwartz, B. S., Ford, P., Bolla, K. I., Agnew, J., \& Bleecker, L. (1991). Solvent-associated olfactory dysfunction: Not a predictor of deficits in learning and memory. American Journal of Psychiatry, 148, $751-756$.
SEMB, G. (1968). The detectability of the odor of butanol. Perception \& Psychophysics, 4, 335-340.

Steinmetz, G., Pryor, G. T., \& Stone, H. (1970). Olfactory adaptation and recovery in man as measured by two psychophysical techniques. Perception \& Psychophysics, 8, 327-330.

ThOMPSON, R. F., \& SPENCER, W. A. (1966). Habituation: A model phenomenon for the study of neuronal substrates of behavior. Psychological Review, 73, 16-43.

Todrank, J., Wysocki, C. J., \& Beauchamp, G. K. (1991). The effects of adaptation on the perception of similar and dissimilar odors. Chemical Senses, 16, 467-482.

Volght, R., \& Atema, J. (1990). Adaptation in chemoreceptor cells. III. Effects of cumulative adaptation. Journal of Comparative Physiology, 166, 865-874.

WANG, H.-W., WysockI, C. J., \& GoLD, G. (1993). Induction of olfactory receptor sensitivity in mice. Science, 260, 998-1000.

Wysocki, C. J., Dorries, K. A., \& Beauchamp, G. K. (1989). Ability to perceive androstenone can be acquired by ostensibly anosmic people. Proceedings of the National Academy of Science, 86, 79767978.

\section{NOTES}

1. Adaptation, as the term is used in this paper, is sufficiently broad to include both sensory adaptation and habituation. Sensory adaptation involves relatively peripheral clianges, whereas habituation involves more central changes. However, the majority of olfactory adaptation experiments do not empirically distinguish these two processes, although both may be influential in the decreased responsiveness to suprathreshold odorants seen following exposure. This distinction is addressed further in the General Discussion.

2. Because the olfactory function of the subjects in these experiments was assessed following exposure to the compound of interest, only comparisons between exposed and unexposed individuals, or across individuals with different levels of exposure, can be used to infer deficits. However, those comparisons assume that the olfactory sensitivity/function of exposed individuals was normal prior to exposure. Such an assumption may not be warranted, if, for instance, deficits in olfactory sensitivity could have allowed certain individuals to remain in jobs in odorous environments.

3. We chose odorants we hoped would be familiar to most subjects in order to avoid the additional variable of stimulus novelty. Future work can (and should) address the effects of odor novelty on adaptation.

4. Studies of odor-exposed individuals, such as worker populations, can document olfactory changes by measuring the degree of deviation in olfactory thresholds or perceived intensity from nonexposed controls.

5. In a preliminary experiment, we verified the perceptual intensity of sample odorant cartridges in several test rooms, where individuals assessed odor intensity three times a week for 3 weeks. No decrements were seen in rated intensity across that time period.

6. Sensitivity to both odorants increased substantially from the first to the second baseline test. Therefore, in comparisons involving baseline versus adaptation and baseline versus recovery we used the final baseline test as a measure of how sensitive the subject was prior to any exposure.

7. There was a significant difference between the first and the second recovery phase test for the adapting odorant only. This suggests that the increase in sensitivity to the control odorant was reaching an asymptote; however, sensitivity was continuing to increase for at least some of the subjects. Indeed, control odorant thresholds could not be measured at the final test for 3 subjects because they could detect the entire range of stimuli available for presentation. These individuals were arbitrarily assigned a threshold value of 25 .

(Manuscript received September 26, 1994; revision accepted for publication September 21, 1995.) 